\title{
Volume preserving centro-affine normal flows
}

\author{
Mohammad N. Ivaki and Alina Stancu
}

\begin{abstract}
We study the long time behavior of the volume preserving $p$-flow in $\mathbb{R}^{n+1}$ for $1 \leq p<\frac{n+1}{n-1}$. By extending Andrews' technique for the flow along the affine normal, we prove that every centrally symmetric solution to the volume preserving $p$-flow converges sequentially to the unit ball in the $C^{\infty}$ topology, modulo the group of special linear transformations.
\end{abstract}

\section{Introduction}

Let $K$ be a compact, centrally symmetric, strictly convex body, smoothly embedded in $\mathbb{R}^{n+1}$. We denote the space of such convex bodies by $\mathcal{K}_{\text {sym }}$. Let

$$
x_{K}: \mathbb{S}^{n} \rightarrow \mathbb{R}^{n+1},
$$

be the Gauss parametrization of $\partial K$, the boundary of $K \in \mathcal{K}_{\text {sym }}$, where the origin of the Euclidean space is chosen to coincide with the center of symmetry of the body. The support function of $\partial K$ is defined by

$$
s_{\partial K}(z):=\left\langle x_{K}(z), z\right\rangle,
$$

for each $z \in \mathbb{S}^{n}$. We denote the matrix of the radii of curvature of $\partial K$ by $\mathfrak{r}=\left[\mathfrak{r}_{i j}\right]_{1 \leq i, j \leq n}$, the entries of $\mathfrak{r}$ considered as functions on the unit sphere too. They are related to the support function by the identity

$$
\mathfrak{r}_{i j}:=\bar{\nabla}_{i} \bar{\nabla}_{j} s+s \bar{g}_{i j},
$$

where $\bar{g}_{i j}$ is the standard metric on $\mathbb{S}^{n}$ and $\bar{\nabla}$ is the standard Levi-Civita connection of $\mathbb{S}^{n}$. We denote the Gauss curvature of $\partial K$ by $\mathcal{K}$ and remark that, as a function on the unit sphere, it is related to the support function of the convex body by

$$
\frac{1}{\mathcal{K}}:=S_{n}=\operatorname{det}\left(\bar{\nabla}_{i} \bar{\nabla}_{j} s+\bar{g}_{i j} s\right):=\frac{\operatorname{det} \mathfrak{r}_{i j}}{\operatorname{det} \bar{g}_{i j}} .
$$

We denote the eigenvalues of $\left[\mathfrak{r}_{i j}\right]_{1 \leq i, j \leq n}$ with respect to the metric $\bar{g}_{i j}$ by $\lambda_{i}$ for $1 \leq i \leq n$. Then the principal curvatures of $\partial K$ are $\kappa_{i}=\frac{1}{\lambda_{i}}$ for $1 \leq i \leq n$. 
For any fixed convex body $K_{0}$ whose interior contains the origin, and whose boundary is of class $C^{2}$ with strictly positive Gauss curvature, the following flow along the centro-affine normal has been defined in [12]:

$$
\frac{\partial}{\partial t} x:=-\mathcal{K}_{0}^{\alpha} \mathcal{N}_{0}, x(\cdot, 0)=x_{K_{0}}(\cdot),
$$

where $\alpha$ is a positive real power which will be made explicit soon, $\mathcal{K}_{0}=$ $\mathcal{K} / s^{n+2}$ is, interestingly, an $S L(n+1)$ invariant called centro-affine curvature and, finally, $\mathcal{N}_{0}=\mathcal{K}_{0}{ }^{-\frac{1}{n+1}}(z)\left(-\mathcal{K}^{\frac{1}{n+1}}(z) z-\bar{\nabla}\left(\mathcal{K}^{\frac{1}{n+1}}(z)\right)\right)$ is the centroaffine normal, both as functions of $z$. The flow was defined for the purpose of finding new global centro-affine invariants of smooth convex bodies in which a certain class of existing invariants arose naturally. Only the short time existence to the flow was then needed. Moreover, several interesting isoperimetric type inequalities were obtained via short-time existence of the flow, [12]. In what follows, we will consider an equivalent (Euclidean) formulation of the flow and, in this paper, we will restrict the power to a certain range.

Let $p \geq 1$ be a fixed real number and let $K_{0} \in \mathcal{K}_{\text {sym }}$. We consider a family of convex bodies $\left\{K_{t}\right\}_{t} \in \mathcal{K}_{\text {sym }}$, and their associated smooth embeddings $x: \mathbb{S}^{n} \times[0, T) \rightarrow \mathbb{R}^{n+1}$, which are evolving according to the $p$-centro-affine flow, namely,

$$
\frac{\partial}{\partial t} x:=-s\left(\frac{\mathcal{K}}{s^{n+2}}\right)^{\frac{p}{n+1+p}} z, \quad x(\cdot, 0)=x_{K_{0}}(\cdot), \quad x(\cdot, t)=x_{K_{t}}(\cdot) .
$$

It is this flow for which we propose the study of asymptotic behavior by applying the techniques of [2]. The long time behavior of the flow in $\mathbb{R}^{2}$ was studied by the first author in [4] using tools of affine differential geometry. It was proved there that the volume preserving $p$-flow with $p \geq 1$ evolves any convex body in $\mathcal{K}_{\text {sym }}$ to the unit disk in Hausdorff distance, modulo $S L(2)$. A further application of the techniques developed in [4] to the $L_{-2}$ Minkowski problem is given in [5]. The case $p=1$, the well-known affine normal flow, was already addressed by Andrews [1,2]. Andrews, investigated the affine normal flow of compact, convex hypersurfaces in any dimension and showed that the volume preserving flow evolves any convex initial bounded open set exponentially fast, in the $C^{\infty}$ topology, to an ellipsoid. In another direction, interesting results for the affine normal flow have been obtained in [8] by Loftin and Tsui regarding ancient solutions, and existence and regularity of solutions on non-compact strictly convex hypersurfaces. 
In this paper, we prove that

Theorem (Main Theorem). Let $1 \leq p<\frac{n+1}{n-1}$ be a real number. Let $x_{K_{0}}$ : $\mathbb{S}^{n} \rightarrow \mathbb{R}^{n+1}$ be a smooth, strictly convex embedding of $K_{0} \in \mathcal{K}_{\mathrm{sym}}$. Then there exists a unique solution $x: \mathbb{S}^{n} \times[0, T) \rightarrow \mathbb{R}^{n+1}$ of equation (1.1) with initial data $x_{K_{0}}$. The rescaled convex bodies given by the embeddings $\left(\frac{V\left(\mathbb{B}^{n+1}\right)}{V\left(K_{t}\right)}\right)^{\frac{1}{n+1}}$ $x_{K_{t}}$ converge sequentially in the $C^{\infty}$ topology to the unit ball, modulo $S L(n+1)$.

The restriction on the range of $p$ is purely technical and it will become evident in Lemma 2.3 which is the only place requiring it.

\section{Uniform lower and upper bound on the principal curvatures}

We will start this section by proving that, under uniform lower and upper bounds on the support function of the evolving convex body, we have uniform lower and upper bounds on the Gauss curvature, which depend only on the dimension $n$, the value of $p$, the bounds on the support function, and time. To obtain the upper bound on the Gauss curvature, we apply a standard technique of Tso [13]. To derive the lower bound bound on the Gauss curvature, we consider the evolution of the dual convex body and we apply Tso's technique to the speed of the dual $p$-flow. This procedure avoids the need for a Harnack estimate, or displacement bounds. In fact, the latter bounds are irrelevant to the $p$-flow as for only $p=1$ is this geometric flow invariant under Euclidean translations.

It is easy to see from $\langle x(z), z\rangle=s(z)$ that, as the convex bodies $\left\{K_{t}\right\}_{t}$ evolve by (1.1), their support functions satisfy the partial differential equation

$$
\partial_{t} s=-s\left(\frac{\mathcal{K}}{s^{n+2}}\right)^{\frac{p}{n+1+p}}
$$

see also [12]. The short time existence and uniqueness of solutions for a smooth and strictly convex initial hypersurface follow from the strict parabolicity of the equation and it was shown in [12]. We will use this latter evolution equation to describe the flow throughout the rest of the paper.

The proofs of the two lemmas pertaining to upper, respectively, lower bound of the Gauss curvature of the evolving convex bodies have similar 
outline yet with some differences. For completeness, we will present both proofs.

Given a convex body $K$, the inner radius of $K, r_{-}(K)$, is the radius of the largest ball contained in $K$; the outer radius of $K, r_{+}(K)$, is the radius of the smallest ball containing $K$. Note that, for any centrally symmetric convex body, the smallest and largest ball as above will be centered at the origin.

Lemma 2.1 (Upper bound on the Gauss curvature). For any smooth, strictly convex solution $\left\{K_{t}\right\}_{\left[0, t_{0}\right]}$ of the evolution equation (2.1) with $0<$ $R_{-} \leq r_{-}\left(K_{t}\right) \leq r_{+}\left(K_{t}\right) \leq R_{+}<+\infty$ for $t \in\left[0, t_{0}\right]$, and some positive numbers $R_{ \pm}$, we have

$$
\mathcal{K}^{\frac{p}{n+p+1}} \leq\left(C+C^{\prime} t^{-\frac{n p}{(n+1)(p+1)}}\right)
$$

where $C$ and $C^{\prime}$ are constants depending on $n, p, R_{-}$and $R_{+}$.

Proof. Let $\alpha:=1-\frac{(n+2) p}{n+1+p}$ and $\beta:=-\frac{p}{n+1+p}$. Consider the function

$$
\Psi=\frac{s^{\alpha} S_{n}^{\beta}}{s-R_{-} / 2}
$$

where $S_{n}$ stands for the $n$th symmetric polynomial in the radii of curvature as a function on the sphere $\mathbb{S}^{n}$. Using the maximum principle, we will show that $\Psi$ is bounded from above by a function of $n, p, R_{-}, R_{+}$, and time. At the point where the maximum of $\Psi$ occurs, we have

$$
0=\bar{\nabla}_{i} \Psi=\bar{\nabla}_{i}\left(\frac{s^{\alpha} S_{n}^{\beta}}{s-R_{-} / 2}\right) \quad \text { and } \quad \bar{\nabla}_{i} \bar{\nabla}_{j} \Psi \leq 0 .
$$

Hence, we obtain

$$
\frac{\bar{\nabla}_{i}\left(s^{\alpha} S_{n}^{\beta}\right)}{s-R_{-} / 2}=\frac{\left(s^{\alpha} S_{n}^{\beta}\right) \bar{\nabla}_{i} s}{\left(s-R_{-} / 2\right)^{2}}
$$

and, consequently,

$$
\bar{\nabla}_{i} \bar{\nabla}_{j}\left(s^{\alpha} S_{n}^{\beta}\right)+\bar{g}_{i j}\left(s^{\alpha} S_{n}^{\beta}\right) \leq \frac{s^{\alpha} S_{n}^{\beta} \mathfrak{r}_{i j}-R_{-} / 2 s^{\alpha} S_{n}^{\beta} \bar{g}_{i j}}{s-R_{-} / 2} .
$$


We calculate

$$
\begin{aligned}
\partial_{t} \Psi= & -\frac{\beta s^{\alpha} S_{n}^{\beta-1}}{s-R_{-} / 2}\left(\dot{S}_{n}\right)_{i j}\left[\bar{\nabla}_{i} \bar{\nabla}_{j}\left(s^{\alpha} S_{n}^{\beta}\right)+\bar{g}_{i j}\left(s^{\alpha} S_{n}^{\beta}\right)\right] \\
& +\frac{S_{n}^{\beta}}{s-R_{-} / 2} \partial_{t} s^{\alpha}+\frac{s^{2 \alpha} S_{n}^{2 \beta}}{\left(s-R_{-} / 2\right)^{2}}
\end{aligned}
$$

where $\left(\dot{S}_{n}\right)_{i j}:=\frac{\partial S_{n}}{\partial \mathfrak{r}_{i j}}$ is the derivative of the $S_{n}$ with respect to the entry $\mathfrak{r}_{i j}$ of the radii of curvature matrix. By Theorem 1, page 102 of [11], applied to the top symmetric polynomial, we have that $\left(\dot{S}_{n}\right)_{i j}$ is a positive definite bilinear form as long as $\partial K$ has positive Gauss curvature at all points. Notice that

$$
\frac{S_{n}^{\beta}}{s-R_{-} / 2} \partial_{t} s^{\alpha}=-\alpha \Psi^{2}+\frac{\alpha R_{-}}{2} \frac{s^{2 \alpha-1} S_{n}^{2 \beta}}{\left(s-R_{-} / 2\right)^{2}} \leq-\alpha \Psi^{2} .
$$

Thus, using inequalities (2.2) and (2.3) we infer that, at the point where the maximum of $\Psi$ is reached, we have

$$
\partial_{t} \Psi \leq \Psi^{2}\left(-n \beta-\alpha+1+\frac{\beta R_{-}}{2} \mathcal{H}\right)
$$

We can control the mean curvature $\mathcal{H}$ from below by a positive power of $\Psi$. First notice that $\mathcal{H} \geq \frac{n}{S_{n}^{\frac{1}{n}}}$. Therefore

$$
\mathcal{H} \geq n\left(\frac{s-R_{-} / 2}{s^{\alpha} S_{n}^{\beta}}\right)^{\frac{1}{n \beta}}\left(\frac{s^{\alpha}}{s-R_{-} / 2}\right)^{\frac{1}{n \beta}} \geq n \Psi^{-\frac{1}{n \beta}}\left(\frac{R_{+}^{\alpha}}{R_{-}-R_{-} / 2}\right)^{\frac{1}{n \beta}}
$$

Therefore, we can rewrite the inequality (2.4) as follows:

$$
\begin{aligned}
\partial_{t} \Psi & \leq \Psi^{2}\left(-n \beta-\alpha+1+\frac{n \beta R_{-}}{2} \Psi^{-\frac{1}{n \beta}}\left(\frac{R_{+}^{\alpha}}{R_{-} / 2}\right)^{\frac{1}{n \beta}}\right) \\
& =-\Psi^{2}\left(C^{\prime}\left(n, p, R_{-}, R_{+}\right) \Psi^{\frac{n+1+p}{n p}}-C(n, p)\right),
\end{aligned}
$$

for positive constants $C(n, p)$ and $C^{\prime}\left(n, p, C, R_{-}, R_{+}\right)$. Hence,

$$
\Psi \leq \max \left\{C\left(n, p, R_{-}, R_{+}\right), C^{\prime}\left(n, p, R_{-}, R_{+}\right) t^{-\frac{n p}{(n+1)(p+1)}}\right\}
$$

for new constants $C$ and $C^{\prime}$. The corresponding claim for $\mathcal{K}$ follows.

Pertaining to the flow by powers of the Gauss curvature, a powerful technique to obtain a uniform lower bound on the Gauss curvatures is using a 
Harnack's inequality and a lower displacement bound [2]. The lower displacement bound controls how much the support of the evolving body decreases depending on time. The displacement bound is obtained by looking at how appropriate barriers, usually balls, with appropriate centers, move along the flow, combined with a containment principle. Despite the fact that we can prove a Harnack's estimate for the $p$-flow, one still needs to obtain the lower displacement bound via barriers. Arbitrarily centered balls are not good barriers in this case because the $p$-flow is not invariant under Euclidean translations and the $p$-flow acting on a ball depends on the choice of the origin. To overcome this difficulty we introduce a new technique. We look at the geometric flow that evolves the dual convex body, the dual $p$-flow.

Let $K^{\circ}$ denote the polar body associated to $K$ with respect to the origin

$$
K^{\circ}=\left\{y \in \mathbb{R}^{n+1} \mid x \cdot y \leq 1, \quad \forall x \in K\right\} .
$$

We will use further the following lemma proved in [12].

Lemma 2.2 (The dual $p$-flow). [12] Let $\left\{K_{t}\right\}_{[0, T)}$ be a smooth, strictly convex solution of the evolution equation (2.1). Then $\left\{K_{t}^{\circ}\right\}_{[0, T)}$ is a solution of the following evolution equation, the expanding p-flow (alternatively called the dual p-flow):

$$
\partial_{t} s^{\circ}=s^{\circ}\left(\frac{\mathcal{K}^{\circ}}{s^{\circ n+2}}\right)^{-\frac{p}{n+1+p}}
$$

It is in next lemma that we need to restrict to the case $p<\frac{n+1}{n-1}$.

Lemma 2.3 (Lower bound on the Gauss curvature). Let $1 \leq p<\frac{n+1}{n-1}$. Assume that $\left\{K_{t}\right\}_{\left[0, t_{0}\right]}$ is a smooth, strictly convex solution of equation (2.1) with $0<R_{-} \leq r_{-}\left(K_{t}\right) \leq r_{+}\left(K_{t}\right) \leq R_{+}<+\infty$ for $t \in\left[0, t_{0}\right]$. Then

$$
\mathcal{K}^{\frac{p}{n+p+1}} \geq \frac{1}{C+C^{\prime} t^{\frac{n p}{(n-1) p-(n+1)}}},
$$

where $C$ and $C^{\prime}$ are constants depending on $n, p, R_{-}$and $R_{+}$.

Proof. Recall from Lemma 2.2 that $\partial_{t} s^{\circ}=s^{\circ}\left(\frac{\mathcal{K}^{\circ}}{s^{\circ+2}}\right)^{-\frac{p}{n+1+p}}$. We define $\alpha:=$ $1+\frac{(n+2) p}{n+1+p}$ and $\beta:=\frac{p}{n+1+p}$. Therefore, the dual flow takes the following form 
$\partial_{t} s^{\circ}=s^{\circ \alpha} S_{n}^{\circ \beta}$. Since $R_{-} \leq r_{-}\left(K_{t}\right) \leq r_{+}\left(K_{t}\right) \leq R_{+}$, we have

$$
\frac{1}{R_{+}} \leq r_{-}\left(K_{t}^{\circ}\right) \leq r_{+}\left(K_{t}^{\circ}\right) \leq \frac{1}{R_{-}} \text {. }
$$

Define

$$
R_{-}^{\circ}:=\frac{1}{R_{+}}, R_{+}^{\circ}:=\frac{1}{R_{-}}
$$

and consider the function

$$
\Phi=\frac{s^{\circ \alpha} S_{n}^{\circ \beta}}{2 R_{+}^{\circ}-s^{\circ}} .
$$

The subsequent computation is carried out at the point where the minimum of $\Phi$ occurs:

$$
0=\bar{\nabla}_{i} \Phi=\bar{\nabla}_{i}\left(\frac{s^{\circ \alpha} S_{n}^{\circ \beta}}{2 R_{+}^{\circ}-s^{\circ}}\right) \quad \text { and } \quad \bar{\nabla}_{i} \bar{\nabla}_{j} \Phi \geq 0
$$

hence we obtain

$$
\frac{\bar{\nabla}_{i}\left(s^{\circ \alpha} S_{n}^{\circ \beta}\right)}{2 R_{+}^{\circ}-s^{\circ}}=-\frac{s^{\circ \alpha} S_{n}^{\circ \beta} \bar{\nabla}_{i} s^{\circ}}{\left(2 R_{+}^{\circ}-s^{\circ}\right)^{2}}
$$

and

$$
\bar{\nabla}_{i} \bar{\nabla}_{j}\left(s^{\circ \alpha} S_{n}^{\circ \beta}\right)+\bar{g}_{i j}\left(s^{\circ \alpha} S_{n}^{\circ \beta}\right) \geq \frac{-s^{\circ \alpha} S_{n}^{\circ \beta} \mathfrak{r}_{i j}^{\circ}+2 R_{+}^{\circ} s^{\circ \alpha} S_{n}^{\circ \beta} \bar{g}_{i j}}{2 R_{+}^{\circ}-s^{\circ}} .
$$

Calculating

$$
\begin{aligned}
\partial_{t} \Phi= & \frac{\beta s^{\circ \alpha} S_{n}^{\circ \beta-1}}{2 R_{+}^{\circ}-s^{\circ}}\left(\dot{S}_{n}^{\circ}\right)_{i j}\left[\bar{\nabla}_{i} \bar{\nabla}_{j}\left(s^{\circ \alpha} S_{n}^{\circ \beta}\right)+\bar{g}_{i j}\left(s^{\circ \alpha} S_{n}^{\circ \beta}\right)\right]+\frac{S_{n}^{\circ \beta}}{2 R_{+}^{\circ}-s^{\circ}} \partial_{t} s^{\circ \alpha} \\
& +\frac{s^{\circ 2 \alpha} S_{n}^{\circ 2 \beta}}{\left(2 R_{+}^{\circ}-s^{\circ}\right)^{2}} .
\end{aligned}
$$

and applying inequality (2.5), we conclude that

$$
\partial_{t} \Phi \geq \Phi^{2}\left(1-n \beta-\alpha+2 \beta R_{+}^{\circ} \mathcal{H}^{\circ}\right)
$$

We now estimate the mean curvature $\mathcal{H}^{\circ}$ from below by a negative power of $\Phi$. As in the proof of the previous lemma, we have

$$
\mathcal{H}^{\circ} \geq n\left(\frac{2 R_{+}^{\circ}-s^{\circ}}{s^{\circ \alpha} S_{n}^{\circ \beta}}\right)^{\frac{1}{n \beta}}\left(\frac{s^{\circ \alpha}}{2 R_{+}^{\circ}-s^{\circ}}\right)^{\frac{1}{n \beta}} \geq n \Phi^{-\frac{1}{n \beta}}\left(\frac{R_{-}^{\circ \alpha}}{2 R_{+}^{\circ}-R_{-}^{\circ}}\right)^{\frac{1}{n \beta}} .
$$


Consequently, inequality (2.6) can be rewritten as follows

$$
\begin{aligned}
\partial_{t} \Phi & \geq \Phi^{2}\left(1-n \beta-\alpha+2 R_{+}^{\circ} n \beta \Phi^{-\frac{1}{n \beta}}\left(\frac{R_{-}^{\circ \alpha}}{2 R_{+}^{\circ}-R_{-}^{\circ}}\right)^{\frac{1}{n \beta}}\right) \\
& =\Phi^{2}\left(-C(n, p)+C^{\prime}\left(n, p, R_{-}^{\circ}, R_{+}^{\circ}\right) \Phi^{-\frac{n+1+p}{n p}}\right)
\end{aligned}
$$

for positive constants $C(n, p)$ and $C^{\prime}\left(n, p, R_{-}^{\circ}, R_{+}^{\circ}\right)$. Hence,

$$
\partial_{t}\left(\frac{1}{\Phi}\right) \leq-C^{\prime}\left(n, p, R_{-}^{\circ}, R_{+}^{\circ}\right)\left(\frac{1}{\Phi}\right)^{\frac{n+1+p}{n p}}+C(n, p)
$$

which implies

$$
\frac{1}{\Phi} \leq \max \left\{C, C^{\prime} t^{\frac{n p}{(n-1) p-(n+1)}}\right\}
$$

for new constants $C$ and $C^{\prime}$. Equivalently, we have a bound for $\Phi$ from below.

Therefore, we have bounded from above $\mathcal{K}^{\circ}$ in terms of $n, p, R_{-}, R_{+}$and time. To complete the proof we recall the following fact: for every $x \in \partial K$, there exists an $x^{\circ} \in \partial K^{\circ}$ such that

$$
\left(\frac{\mathcal{K}}{s^{n+2}}\right)(x)\left(\frac{\mathcal{K}^{\circ}}{s^{\circ n+2}}\right)\left(x^{\circ}\right)=1
$$

where $x$ and $x^{\circ}$ are related by $\left\langle x, x^{\circ}\right\rangle=1$, with $\langle$,$\rangle the inner product in$ $\mathbb{R}^{n+1}$. A proof of this identity in the smooth setting is simple. A proof in a more general non-smooth setting can be found in [3], see also [9]. For a non-smooth convex body one needs to replace $\mathcal{K}$ with the generalized Gauss curvature, the identity then holds almost everywhere with respect to the Hausdorff measure on $\partial K$.

By the above identity, we conclude that $\mathcal{K}$ is bounded from below by constants depending on $n, p, R_{-}, R_{+}$and time.

We point out that in concluding the long time existence of solutions, and asymptotic behavior, to the flow in $\mathbb{R}^{2}$, the following two lemmas in this section are not necessary.

Lemma 2.4 (Lower bound on the principal curvatures). Assume that $n>1$. Let $\left\{K_{t}\right\}_{\left[0, t_{0}\right]}$ be a smooth strictly convex solution of equation (2.1) with 
$0<R_{-} \leq r_{-}\left(K_{t}\right) \leq r_{+}\left(K_{t}\right) \leq R_{+}<+\infty$ and suppose that

$$
C_{1} \leq S_{n} \leq C_{2}
$$

for all $t \in\left[0, t_{0}\right]$. Then there exist constants $C$ and $C^{\prime}$ depending on $n, p, R_{-}$, $R_{+}, C_{1}$ and $C_{2}$ such that

$$
\frac{1}{\kappa_{i}} \leq\left(C+C^{\prime} t^{-(n-1)}\right)
$$

for all $t \in\left[0, t_{0}\right]$.

Proof. We first compute the evolution equation of $\mathfrak{r}_{i j}=\bar{\nabla}_{i} \bar{\nabla}_{j} s+s \bar{g}_{i j}$ for which a similar computation has been carried in [2].

Set $\alpha:=-1+\frac{(n+2) p}{n+1+p}$ and $\beta:=\frac{p}{n+1+p}$.

$$
\begin{aligned}
\partial_{t} \mathfrak{r}_{i j}= & \beta s^{-\alpha} S_{n}^{-(1+\beta)}\left(\dot{S}_{n}\right)_{k l} \bar{\nabla}_{k} \bar{\nabla}_{l} \mathfrak{r}_{i j}-\beta(\beta+1) s^{-\alpha} S_{n}^{-(2+\beta)} \bar{\nabla}_{i} S_{n} \bar{\nabla}_{j} S_{n} \\
& +\beta s^{-\alpha} S_{n}^{-(1+\beta)}\left(\ddot{S}_{n}\right)_{k l ; m n} \bar{\nabla}_{i} \mathfrak{r}_{k l} \bar{\nabla}_{j} \mathfrak{r}_{m n} \\
& +(n \beta-1) s^{-\alpha} S_{n}^{-\beta} \bar{g}_{i j}-\beta s^{-\alpha} S_{n}^{-(1+\beta)}\left(\dot{S}_{n}\right)_{k l} \mathfrak{r}_{i j} \bar{g}_{k l} \\
& +S_{n}^{-\beta} \bar{\nabla}_{i} \bar{\nabla}_{j} s^{-\alpha}+\beta S_{n}^{-(1+\beta)} \bar{\nabla}_{i} s^{-\alpha} \bar{\nabla}_{j} S_{n}+\beta S_{n}^{-(1+\beta)} \bar{\nabla}_{j} s^{-\alpha} \bar{\nabla}_{i} S_{n}
\end{aligned}
$$

a: Estimating the terms on the first line: The first term on the first line is an essential good term viewed as an elliptic operator which is nonpositive at the point and direction where the maximum of $\mathfrak{r}_{i j}$ occurs. The second term is an essential good negative term.

b: Estimating the term on the second line: Concavity of $S_{n}^{\frac{1}{n}}$, see again [11], gives

$$
\left[\left(\ddot{S}_{n}\right)_{k l ; m n}-\frac{n-1}{n S_{n}}\left(\dot{S}_{n}\right)_{k l}\left(\dot{S}_{n}\right)_{m n}\right] \bar{\nabla}_{i} \mathfrak{r}_{k l} \bar{\nabla}_{j} \mathfrak{r}_{m n} \leq 0 .
$$

c: Estimating the terms on the last line:

$$
\begin{aligned}
\bar{\nabla}_{i} \bar{\nabla}_{j} s^{-\alpha} & =-\alpha \frac{\bar{\nabla}_{i} \bar{\nabla}_{j} s}{s^{\alpha+1}}+\alpha(\alpha+1) \frac{\bar{\nabla}_{i} s \bar{\nabla}_{j} s}{s^{\alpha+2}} \\
& =-\alpha \frac{\left(\mathfrak{r}_{i j}-\bar{g}_{i j} s\right)}{s^{\alpha+1}}+\alpha(\alpha+1) \frac{\bar{\nabla}_{i} s \bar{\nabla}_{j} s}{s^{\alpha+2}}
\end{aligned}
$$

This gives

$$
S_{n}^{-\beta} \bar{\nabla}_{i} \bar{\nabla}_{j} s^{-\alpha} \leq-\alpha C S_{n}^{-\beta} \mathfrak{r}_{i j}+\alpha C^{\prime} S_{n}^{-\beta} \bar{g}_{i j} \leq \alpha C^{\prime \prime} S_{n}^{-\beta} \bar{g}_{i j},
$$


where we used the boundedness of $\bar{\nabla}_{i} s$ from above and the assumptions of the lemma. Notice that $|x|^{2}=s^{2}+|\bar{\nabla} s|^{2}$. Therefore, as $s$ is bounded, $|\bar{\nabla} s|$ must also be bounded. Here we used $|\cdot|$ for the Euclidean norm in $\mathbb{R}^{n+1}$

The other term on the last line can be estimated by Young's inequality:

$$
\left|\bar{\nabla}_{i} s^{-\alpha} \bar{\nabla}_{j} S_{n}\right| \leq C \varepsilon\left|\bar{\nabla}_{j} S_{n}\right|^{2}+C \varepsilon^{-1}
$$

Combining inequality (2.7) and equations (2.8) and (2.9), for $\varepsilon$ small enough, we have

$$
\partial_{t} \mathfrak{r}_{i j} \leq \beta s^{-\alpha} S_{n}^{-(1+\beta)}\left(\dot{S}_{n}\right)_{k l} \bar{\nabla}_{k} \bar{\nabla}_{l} \mathfrak{r}_{i j}+C^{\prime} S_{n}^{-\beta} \bar{g}_{i j}-\beta C S_{n}^{-(1+\beta)}\left(\dot{S}_{n}\right)_{k l} \mathfrak{r}_{i j} \bar{g}_{k l}
$$

Therefore, the maximum of the hypersurface's $\mathfrak{r}_{i j}$, as a function of time, satisfies

$$
\partial_{t}\left(\mathfrak{r}_{i j}\right)_{\max } \leq C S_{n}^{-\beta}\left(\bar{g}_{i j}-C S_{n}^{-\frac{n}{n-1}}\left(\mathfrak{r}_{i j}\right)_{\max }^{\frac{n}{n-1}}\right)
$$

This implies that, for $\left(\mathfrak{r}_{i j}\right)_{\max }$ very large, the quantity in parentheses is negative, while $S_{n}$ is bounded away from zero, hence the behavior of $\left(\mathfrak{r}_{i j}\right)_{\max }$ when large is modeled by the differential inequality

$$
\frac{d\left(\mathfrak{r}_{i j}\right)_{\max }}{d t} \leq-C\left(\mathfrak{r}_{i j}\right)_{\max }^{\frac{n}{n-1}}
$$

We thus conclude that

$$
\max _{i, j}\left(\mathfrak{r}_{i j}\right)_{\max } \leq C+C^{\prime} t^{-(n-1)}
$$

for some positive constants $C, C^{\prime}$. As, for any real symmetric matrix $A$, its highest eigenvalue is $\lambda_{\max }(A)=\sup _{u \in \mathbb{R}^{n},\|u\|=1}|\langle u, A u\rangle|$, we obtain the upper bound on the highest radius of curvature of the form $C+C^{\prime} t^{-(n-1)}$, where the constants have been redenoted the same for simplicity.

Lemma 2.5 (Lower and upper bounds on the principal curvatures). Assume that $n>1$. Let $\left\{K_{t}\right\}_{\left[0, t_{0}\right]}$ be a smooth, strictly convex solution of equation 
(2.1) with $0<R_{-} \leq r_{-}\left(K_{t}\right) \leq r_{+}\left(K_{t}\right) \leq R_{+}<+\infty$ and

$$
C_{1} \leq S_{n} \leq C_{2}
$$

for all $t \in\left[0, t_{0}\right]$. Then there exist constants $C_{3}, C_{3}^{\prime}, C_{4}$ and $C_{4}^{\prime}$ depending on $n, p, R_{-}, R_{+}, C_{1}$ and $C_{2}$ such that, $\forall t \in\left[0, t_{0}\right]$,

$$
\frac{1}{C_{3}+C_{4} t^{-(n-1)}} \leq \kappa_{i} \leq\left(C_{3}^{\prime}+C_{4}^{\prime} t^{-(n-1)}\right)^{n-1} .
$$

Proof. The lower bound on the principal curvatures has been, in fact, established in Lemma 2.4. Consequently, we also obtain now the upper bound as the product of the principal curvatures is bounded from above. Suppose that $\kappa_{1} \geq \kappa_{2} \geq \cdots \geq \kappa_{n}$, then

$$
C_{1}^{-1} \geq \mathcal{K}=\Pi_{i=1}^{n} \kappa_{i}=\kappa_{1} \cdot \Pi_{i=1}^{n-1} \kappa_{i} \geq \kappa_{1}\left(C_{3}+C_{4} t^{-(n-1)}\right)^{-(n-1)} .
$$

Theorem 2.6. Let $1 \leq p<\frac{n+1}{n-1}$ be a real number. Let $x_{K_{0}}: \mathbb{S}^{n} \rightarrow \mathbb{R}^{n+1}$ be a smooth, strictly convex embedding of $K_{0} \in \mathcal{K}_{\mathrm{sym}}$. Then there exists a unique solution $x: \mathbb{S}^{n} \times[0, T) \rightarrow \mathbb{R}^{n+1}$ of equation (1.1) with initial data $x_{K_{0}}$, for a maximal finite $T$, such that $\lim _{t \rightarrow T} V\left(K_{t}\right)=0$.

Proof. Suppose that $S_{0}$ is a sphere which, at time zero, encloses $K_{0}$. It is clear that, by applying the $p$-flow to $S_{0}$, the evolving spheres $S_{t}$ converge to a point in finite time. By a comparison principle, $K_{t}$ remains in the closure of $S_{t}$, therefore $T$ must be finite. Suppose now that $V\left(K_{t}\right)$ does not tend to zero. Then, we must have $s \geq r$, for some $r>0$ on $[0, T)$. By Lemmas 2.1, 2.3, 2.4 and 2.5 the principal curvatures of the solution remains uniformly bounded on $[0, T)$ from below and above. Consequently the evolution equation (1.1) is uniformly parabolic on $[0, T)$, and bounds on higher derivatives of the support function follows by [7] and Schauder theory. Hence, we can extend the solution after time $T$, contradicting its definition.

\section{Convergence of the volume preserving $p$-flow}

In this section, we will conclude the proof of the main theorem.

We will start with a few facts on convex bodies. For a given convex body $K$, we call the volume of $K$ its Lebesgue measure as a subset of $\mathbb{R}^{n+1}$. 
A celebrated affine invariant quantity associated to $K$ is its affine surface area which for a body with a smooth boundary can be expressed by

$$
\Omega(K)=\int_{\mathbb{S}^{n}} \mathcal{K}^{-\frac{n+1}{n+2}} d \mu_{\mathbb{S}^{n}}
$$

The affine surface area satisfies an isoperimetric inequality which we will state soon in a more general setting. The extension of the affine surface area defined by Lutwak [10] for $p \geq 1$ in the context of the Firey-BrunnMinkowski theory of convex bodies, called the $L_{p}$-affine or $p$-affine surface area, is

$$
\Omega_{p}(K)=\int_{\mathbb{S}^{n}} \frac{s}{\mathcal{K}}\left(\frac{\mathcal{K}}{s^{n+2}}\right)^{\frac{p}{n+1+p}} d \mu_{\mathbb{S}^{n}}
$$

which reduces to the usual affine surface area for $p=1$. At the core of the centro-affine geometry, lies the $p$-affine isoperimetric inequality due to Lutwak, $p \geq 1$, which generalizes the classical $p=1$ case,

$$
\frac{\Omega_{p}^{n+p+1}(K)}{V^{n-p+1}(K)} \leq(n+1)^{n+p+1} \omega_{n+1}^{2 p},
$$

where $\omega_{n+1}=V\left(\mathbb{B}^{n+1}\right)$ is the volume of the unit ball in $\mathbb{R}^{n+1}$, [10]. The equality holds above if and only if $\mathrm{K}$ is a centered ellipsoid.

The following result follows directly from the inequality of Proposition 4.2 in $[12]$.

Theorem (Monotonicity of the $p$-affine isoperimetric ratio). Let $\left\{K_{t}\right\}_{[0, T)}$ be a smooth, strictly convex solution of equation (2.1). Then the p-affine isoperimetric ratio, $\frac{\Omega_{p}^{n+1+p}\left(K_{t}\right)}{V^{n+1-p}\left(K_{t}\right)}$, is non-decreasing along the $p$-flow. The monotonicity is strict unless $K_{t}$ is an ellipsoid centered at the origin.

Finally, we recall also the following famous theorem by Fritz John.

Theorem (John's Lemma [6]). Let $K$ be a convex body in $\mathbb{R}^{n+1}$. Then there exist absolute constants $c$ and $C$, and an affine linear transformation $L \in S L(n+1)$ such that

$$
c \leq\left(\frac{V\left(\mathbb{B}^{n+1}\right)}{V(K)}\right)^{\frac{1}{n+1}} s_{L(K)} \leq C,
$$

where $\mathbb{B}^{n+1}$ denotes the unit ball in $\mathbb{R}^{n+1}$. 
Let now $x: \mathbb{S}^{n} \times[0, T] \rightarrow \mathbb{R}^{n+1}$ be a solution of equation (1.1). Then for each $\lambda>0$, note that $x_{\lambda}$ defined by $x_{\lambda}: \mathbb{S}^{n} \times\left[0, \lambda^{\frac{(2 n+2) p}{n+1+p}} T\right] \rightarrow \mathbb{R}^{n+1}$ with

$$
x_{\lambda}(\theta, t)=\lambda x\left(\theta, \lambda^{-\frac{(2 n+2) p}{n+1+p}} t\right)
$$

is also a solution of evolution equation (1.1).

Proof of the main theorem: We follow the procedure in [2]. Fix $t \in[0, T)$. Define $\tilde{s}$ a solution of (2.1), by the rescaling property, as follows

$$
\tilde{s}(z, \tau)=\left(\frac{V\left(\mathbb{B}^{n+1}\right)}{V\left(K_{t}\right)}\right)^{\frac{1}{n+1}} s\left(z, t+\left(\frac{V\left(\mathbb{B}^{n+1}\right)}{V\left(K_{t}\right)}\right)^{-\frac{2 p}{n+1+p}} \tau\right),
$$

where $\tilde{s}(\cdot, 0)$ is the support function of $\left(\frac{V\left(\mathbb{B}^{n+1}\right)}{V\left(K_{t}\right)}\right)^{\frac{1}{n+1}} L_{t} K_{t}$ and $L_{t} \in S L(n+$ 1 ) is obtained from John's Lemma for the convex body $K_{t}$. Therefore,

$$
c \leq \tilde{s}(z, 0) \leq C .
$$

Let $B_{r}$ denote the ball of radius $r$ centered at the origin. Thus, $B_{c}$ is contained in the convex body associated with the support function $\tilde{s}(\cdot, 0)$. The containment principle, see for example Proposition 2.2 in [12], insures that $B_{c / 2}$ will be contained in the convex body associated with the support function $\tilde{s}(\cdot, \tau)$, for $\tau \in[0, \delta]$, where $\delta$ is the time that $B_{c}$ becomes $B_{c / 2}$ under the $p$-flow. This time can be found explicitly as the evolution of a ball of radius $\rho$ centered at the origin is $\rho_{t}=-\rho^{(n+1-(2 n+1) p) /(n+p+1)}$. Now Lemmas 2.1, 2.3, 2.4 and 2.5 imply that there are uniform lower and upper bounds on the principal curvatures and on the speed of the flow on the time interval $[\delta / 2, \delta]$. Therefore, by [7], we conclude that there are uniform bounds on higher derivatives of the curvature. Consequently, all quantities related to the original solution that are both scaling invariant and invariant under $S L(n+1)$ satisfy uniform bounds on the time interval $\left[t+\frac{C}{2} V\left(K_{t}\right)^{\frac{2 p}{n+1+p}}, t+C V\left(K_{t}\right)^{\frac{2 p}{n+1+p}}\right]$. Since $t$ is arbitrary and $C$ is an absolute constant, we have uniform bounds on the time interval $[T / 2, T)$. It means that all the affine invariant quantities of the normalized solution to the $p$-flow are uniformly bounded on the time interval $[T / 2, T)$. We point out here that if $n=1$, only Lemma 2.1 and Lemma 2.3 are needed to derive such uniform bounds on the time interval $[T / 2, T)$.

Consequently, there is a sequence of times $\left\{t_{k}\right\}_{k \in \mathbb{N}}$ such that $t_{k}$ approaches $T$ and $L_{t_{k}}\left(K_{t_{k}}\right)$ converges in the $C^{\infty}$ topology to a convex body 
$\tilde{K}_{T}$. Now monotonicity of the $p$-affine isoperimetric ratio and Theorem 2.6 with a similar argument as in [2], implies that $\tilde{K}_{T}$ must be an ellipsoid. Therefore,

$$
\lim _{t_{k} \rightarrow T} \frac{\Omega_{p}^{n+1+p}\left(K_{t_{k}}\right)}{V^{n+1-p}\left(K_{t_{k}}\right)}=(n+1)^{n+1+p} \omega_{n+1}^{2 p},
$$

and again by monotonicity of the $p$-affine isoperimetric ratio

$$
\lim _{t \rightarrow T} \frac{\Omega_{p}^{n+1+p}\left(K_{t}\right)}{V^{n+1-p}\left(K_{t}\right)}=(n+1)^{n+1+p} \omega_{n+1}^{2 p} .
$$

From the equality case in the $p$-affine isoperimetric inequality [10], it follows that, modulo $S L(n+1)$,

$$
\lim _{t \rightarrow T}\left(\frac{V\left(\mathbb{B}^{n+1}\right)}{V\left(K_{t}\right)}\right)^{\frac{1}{n+1}} K_{t}=\mathbb{B}^{n+1}
$$

sequentially in the $C^{\infty}$ topology. The proof is complete.

\section{Acknowledgment}

We would like to thank the referees for their helpful comments and the thorough attention given to our manuscript.

\section{References}

[1] B. Andrews, Contraction of convex hypersurfaces by their affine normal, J. Differ. Geom. 43 (1996), 207-230.

[2] B. Andrews, Motion of hypersurfaces by Gauss curvature, Pacific J. Math. 195 (2000), 1-34.

[3] D. Hug, Curvature relations and affine surface area for a general convex body and its polar, Results Math. 29 (1996), 233-248.

[4] M.N. Ivaki, Centro-affine curvature flows on centrally symmetric convex curves, to appear in Trans. Amer. Math. Soc. arXiv:1205.6456v2 [math.DG].

[5] M.N. Ivaki, A flow approach to the $L_{-2}$ Minkowski problem, Adv. Appl. Math. 50 (2012), 445-464; doi: http://dx.doi.org/10.1016/ j.aam.2012.09.003. 
[6] F. John, Extremum problems with inequalities as subsidiary conditions, 187-204 in Studies and essays presented to R. Courant on his 60th Birthday (8 January 1948), Interscience, New York, 1948.

[7] N.V. Krylov, Nonlinear elliptic and parabolic equations of the second order, D. Reidel Publishing Co., Dordrecht, 1987.

[8] J. Loftin and M.P. Tsui, Ancient solutions of the affine normal flow, J. Differ. Geom. 78 (2008), 113-162.

[9] M. Ludwig and M. Reitzner, A classification of $S L(n)$ invariant valuations, Ann. Math. 172 (2010), 1223-1271.

[10] E. Lutwak, The Brunn-Minkowski-Fiery theory II: Affine and geominimal surface areas, Adv. Math. 118 (1996), 244-294.

[11] D.S. Mitrinowic, Analytic inequalities, Springer-Verlag, BerlinHeidelberg-New York, 1970.

[12] A. Stancu, Centro-affine invariants for smooth convex bodies, Int. Math. Res. Not. IMRN, 2012 (2012), 2289-2320; doi: 10.1093/imrn/rnr110.

[13] K. Tso, Deforming a hypersurface by its Gauss-Kronecker curvature, Comm. Pure Appl. Math. 38 (1985), 867-882.

Department of Mathematics and Statistics

ConCordia University

Montreal, QC

CANADA, H3G 1M8

E-mail address: mivaki@mathstat.concordia.ca

E-mail address: stancu@mathstat.concordia.ca

ReCeived September 27, 2012 